\title{
Archives
}

\section{L’Association des archivistes du Québec à travers " ses " histoires}

\section{Louise Gagnon-Arguin}

Volume 48, numéro 1, 2019

$46^{\mathrm{e}}$ Congrès de l'Association des archivistes du Québec

URI : https://id.erudit.org/iderudit/1060814ar

DOI : https://doi.org/10.7202/1060814ar

Aller au sommaire du numéro

Éditeur(s)

Association des archivistes du Québec (AAQ)

ISSN

0044-9423 (imprimé)

2369-9256 (numérique)

Découvrir la revue

Citer cet article

Gagnon-Arguin, L. (2019). L’Association des archivistes du Québec à travers « ses » histoires. Archives, 48(1), 41-53. https://doi.org/10.7202/1060814ar
Résumé de l'article

À partir d'une approche historiographique, cet article reprend les différents textes ou publications qui ont porté sur l'histoire de l'Association des archivistes du Québec (AAQ), de 1974 à nos jours, en soulignant les grandes lignes de leur contenu. Il signale, entre autres, les articles écrits à l'occasion des anniversaires de la fondation de l'Association et présentés particulièrement dans la revue Archives. Il mentionne aussi les textes publiés dans d'autres revues québécoises ou étrangères. En faisant cette synthèse, l'article veut témoigner de l'évolution de l'AAQ et de ses préoccupations à travers le temps. 


\section{6 ${ }^{\mathrm{E}}$ CONGRÈS DE L'AAQ}

\section{L'Association des archivistes du Québec à travers « ses » histoires}

\section{LOUISE GAGNON-ARGUIN, PH. D.}

Professeure associée, École de bibliothéconomie et des sciences de I'information (EBSI), Université de Montréal

«L'histoire doit maintenir vivante la vie » (Americo Castro).

Depuis 1967, I'histoire de I'Association des archivistes du Québec (AAQ) a fait l'objet de près de vingt articles parus principalement dans la revue Archives, mais aussi dans d'autres publications liées aux domaines des archives et de l'histoire. Sous des angles divers, leurs auteurs ont fait ressortir des aspects de la vie de l'Association en prenant pour prétexte différents moments de sa trajectoire.

À l'occasion du 50 anniversaire de l'Association, au lieu de tenter de réécrire son histoire, nous rappellerons plutôt les contributions déjà publiées en les situant dans le temps et par rapport à différentes thématiques. Après avoir rappelé les articles produits à l'occasion des anniversaires de l'Association et en avoir présenté un résumé, nous reprendrons certaines thématiques qui découlent des sujets de ces articles. II s'agit donc, en somme, d'une revue de la littérature sur I'histoire de I'AAQ. 


\section{UNE PREMIÈRE HISTOIRE DE L'AAQ, 1974}

Un texte publié en 1974 dans la revue Archives et écrit par Robert Garon constitue la première histoire publiée de I'Association. L'auteur présente I'AAQ comme ayant "I'honneur d'être la plus ancienne association indépendante d'archivistes au Canada et celui de regrouper le plus grand nombre de membres» (Garon, 1974, p. 6) (300 à cette date). II en rappelle les origines, les difficultés et les échecs inhérents à la mise en place d'une telle structure associative. II en présente les buts et les objectifs en soulignant particulièrement celui de participer au perfectionnement de ses membres; à ce titre, il signale quelques réalisations comme l'organisation de cours de formation et la création de publications comme la revue Archives. Rappelant de plus que toute personne œuvrant dans un service d'archives ou dans un service de gestion de documents peut devenir membre de I'Association, qu'elle travaille dans I'un ou l'autre de ces secteurs. II y voit une richesse professionnelle pour les uns et pour les autres. Ce texte se conclut par un appel à des échanges avec des professionnels en dehors du Québec en vue d'un partage d'expériences.

\section{LES OCCASIONS D'ÉCRIRE: LES ANNIVERSAIRES}

Les anniversaires d'une organisation servent souvent d'aiguillon pour «se rappeler». Différents auteurs ont profité de ces occasions pour faire le point sur la situation de l'Association, de ses activités et de ses réalisations.

\subsection{0 ans, 1978}

Un numéro de la revue Archives, qui constitue les actes du VII congrès de l'Association tenu à Hull (Gatineau) les 18 et 19 mai 1978, présente trois textes sous le titre «Bilan et perspective». Sans pour autant signaler de façon explicite le $10^{\mathrm{e}}$ anniversaire de l'AAQ, chaque auteur (Garon, Minotto et Couture, 1978) se situe dans ce contexte. Robert Garon identifie deux moments forts de ces dix ans: la fondation et la mise en place de l'organisation proprement dite, dont la création de la revue Archives et du bulletin d'information La Chronique, et la création de deux sections à l'intérieur de l'organisation: la section Archives historiques et la section Gestion des documents. Cette dernière suscitant la controverse dans le milieu, l'auteur conclut en soulignant l'importance des échanges 
et de l'ouverture entre les différents milieux de pratique. Claude Minotto voit dans l'Association un lieu de discussion professionnelle qui va au-delà des institutions comme lieu de travail. II lance lui aussi un appel à des échanges entre les professionnels archivistes et les gestionnaires de documents. Quant à Carol Couture, il subdivise déjà l'histoire de la jeune Association en trois périodes: la découverte, de 1968 à 1972, qualifiée de «lune de miel» entre l'AAQ et ses membres; le perfectionnement, de 1969 à 1975, où il perçoit un besoin d'acquisition de connaissances manifeste chez les membres; et la recherche, de 1975 et au-delà qui devrait se traduire par la «poursuite d'une fin positive... et d'un idéal commun» sous-jacent, soit la collaboration entre les archivistes et les gestionnaires de documents (Couture, 1978).

\subsection{5 ans, 1982}

À I'occasion des 15 ans de I'Association, un numéro spécial du bulletin La Chronique paraît en 1982 sous le titre « Félicitations à notre Association pour son $15^{\mathrm{e}}$ anniversaire » (Choquette, 1982). II comprend trois textes. Un premier rappelle les grands événements de la fondation et reprend à cet effet les comptes rendus des réunions des 30 octobre, 20 novembre et 9 décembre 1967. Ce numéro contient aussi deux témoignages d'archivistes fondateurs. François Beaudin signe le premier sous un titre révélateur: "Conçu à Santa Fe... et né à Québec ». II reprend le récit des événements ayant mené à l'idée de mettre en place une association d'archivistes. Puis Claude Lessard se remémore, avec une certaine nostalgie, les bons moments des premières réunions générales et l'atmosphère qui y régnait. II souligne aussi l'importance de la revue Archives et de sa parution régulière, et s'inquiète de la situation financière fragile de I'AAQ.

\subsection{0 ans de congrès}

Rappelant les vingt premiers congrès de l'Association - 1972-1991 -, Claude Lessard effectue un survol de ces événements (Lessard, 1991). Pour chaque congrès, il énumère le lieu et les dates de sa tenue et signale les thèmes qui le structuraient. II rappelle la répartition des programmes (conférences, activités sociales), déplore l'absence de chiffres quant au nombre de participants tout en signalant la présence de nombreux religieux aux premiers congrès. II signale aussi quelques faits marquants 
de certains congrès (présentation des armoiries de I'AAQ, premier congrès à se tenir dans un hôtel, etc.). II s'agit d'une synthèse riche en informations sur ces premiers congrès.

\subsection{5 ans, 1992}

Cet anniversaire coïncide avec la tenue du XII congrès international des archives à Montréal en 1992. À cette occasion, un numéro spécial de la revue Archives (vol. 24, $\left.n^{\text {os }} 1-2,1992\right)$ présente un panorama de «L'expertise québécoise». Les thèmes abordés sont variés, allant de I'histoire de I'Association à celle des Archives nationales du Canada, en passant par la présentation des lois québécoises sur les archives et sur l'accès à l'information et la protection des renseignements personnels, ainsi que de quelques autres ayant un impact sur les archives. Plusieurs articles exposent la situation des archives dans divers milieux, le tout étant précédé de la présentation du Code d'éthique de l'Association et des valeurs professionnelles qui ont conduit à son élaboration.

L'article sur l'histoire de l'Association (Gagnon-Arguin, 1992b) rappelle les principaux événements qui ont marqué les années 1967 à 1988. Il étudie l'évolution de ses objectifs sur ces vingt années, son organisation administrative, ses effectifs, son financement, les différents services à ses membres, ses rôles politique et social, et sa place dans les contextes canadien et international. II soulève aussi les difficultés relatives à la représentativité de l'Association et les tensions qui ont marqué la relation entre les "archivistes» et les "gestionnaires de documents», comme on disait à l'époque, allant même jusqu'à remettre en question le nom même de l'AAQ. Dans un texte sur le même sujet publié dans son ouvrage L'archivistique, son histoire, ses acteurs depuis 1960, l'auteure ajoute à l'histoire de l'Association l'étude de la revue Archives, le sujet des articles, de leurs auteurs et des comptes rendus qu'on y retrouve (Gagnon-Arguin, 1992a).

\subsection{0 ans, 2007}

Le $40^{\mathrm{e}}$ anniversaire de la fondation de l'Association a été l'occasion de produire un numéro spécial de la revue Archives (vol. 39, n 1). Trois articles revisitent, chacun à leur façon, différents volets de son histoire. 
L'article de Louis Garon couvre l'histoire de l'AAQ de 1988 à 2006 (Garon, 2007-2008, p. 87). II présente les actions relatives à la promotion de l'archivistique et des archives à travers les actions politiques et les publications. Par la définition des tâches, le code de déontologie, la certification, la formation et le perfectionnement, il circonscrit la promotion de la reconnaissance professionnelle. II fait aussi état du fonctionnement de l'Association par ses diverses structures. II termine enfin par une présentation de la Déclaration québécoise sur les archives et conclut que le développement de l'AAQ repose sur le degré d'engagement élevé de sa direction et de plusieurs de ses membres malgré des moyens limités.

Diane Baillargeon et Michel Lévesque dressent une rétrospective des mémoires présentés par l'AAQ lors de l'étude de plusieurs projets de loi par l'Assemblée nationale du Québec et par le Parlement fédéral, ainsi que différentes prises de position de l'AAQ de 1992 à 2007 (Baillargeon et Lévesque, 2007-2008). Ils signalent en premier lieu le rôle du Comité des affaires professionnelles comme cheville ouvrière du travail de représentation ainsi que la création du poste de responsable des Affaires législatives. Sous différents thèmes, ils font ensuite état des interventions de l'AAQ pour promouvoir l'archivistique, et de la place des archives et des archivistes dans la culture et le patrimoine. En terminant, ils affirment que «... toutes ces interventions constituent une synthèse historique qui met en évidence la vision et l'engagement de celles et ceux qui s'impliquent au sein de I'Association» (Baillargeon et Lévesque, 2007-2008, p. 87). La bibliographie qui complète l'article présente notamment une liste des mémoires présentés entre 1993 et 2005.

Robert Nahuet propose un survol des différentes activités de formation et de perfectionnement tenues depuis la fondation de l'AAQ (Nahuet, 2007-2008). Rappelant les premières formations dispensées par des archivistes d'expérience à Cap-Rouge et à Montréal entre 1968 à 1973, il présente par la suite les programmes de formation institutionnels, dont ceux des universités qui débutent en 1983. Au titre de la formation offerte par l'Association, il rappelle les quelque 400 conférences prononcées dans le cadre des congrès annuels entre 1968 et 1988 . De plus, sous la responsabilité de différents comités mis sur pied par l'Association et chargés d'élaborer un programme de perfectionnement pour ses membres, un grand nombre d'activités se sont tenues tant dans les sections régionales qu'à l'occasion de congrès. L'auteur dresse la liste des 
activités offertes par l'Association entre 1977 et 1988, puis les activités subventionnées tenues entre 1994 et 2005, en précisant les modalités de leur présentation et les personnes impliquées dans leur organisation. II fait aussi une place à ces autres outils de formation que sont les publications. Selon Nahuet, «... les activités de perfectionnement de I'AAQ... [sont] indispensables à la mise à jour des connaissances (théoriques et pratiques) des spécialistes de la gestion de l'information organique et consignée [...] la professionnalisation des archivistes passe par une formation continue et permanente»(Nahuet, 2007-2008, p. 110).

\subsection{0 ans, 2017}

Qu'est-ce qui a été écrit à l'occasion du $50^{\mathrm{e}}$ anniversaire de la fondation de l'Association? Déjà, quelques réalisations sont dignes de mention.

Original dans son approche, un article de Catherine Dugas présente les cinquante ans de l'AAQ à travers la participation du personnel de la Division de la gestion des documents et des archives de I'Université de Montréal à la vie de l'Association (Dugas, 2017). Elle rappelle en détail le rôle joué par chacun des membres du personnel de la Division à l'un ou l'autre des moments de la vie de l'AAQ: les débuts de 1967 à 1977, l'adolescence de 1980 à 1992, le présent des années 1990 à nos jours. Cet article retrace une certaine histoire de l'AAQ à travers le vécu de quelques personnes d'un milieu de travail qui ont participé de façon très active au développement et à l'engagement de I'AAQ. N'oublions pas que l'heureux prétexte derrière cet article est la coïncidence entre le $50^{\mathrm{e}}$ anniversaire de l'AAQ et celui de la Division de la gestion des documents et des archives de l'Université de Montréal.

Une Ligne du temps expose sur le site Internet de I'Association les principaux événements qui ont marqué la vie de I'AAQ. Cette chronologie est regroupée autour de cinq thèmes, soit l'AAQ, vie associative et soutien à la profession, I'AAQ et le développement de la discipline archivistique, I'AAQ et la politique canadienne et québécoise, l'AAQ et l'environnement culturel et social, et l'AAQ sur le plan international. Préparée à partir de différentes sources, dont le bulletin La Chronique et I'Infolettre, cette Ligne du temps récapitule les événements qui se sont produits au cours des cinquante années de l'Association. 
Somme toute, I'Association a fait l'objet de plusieurs analyses et a été étudiée sous différents angles au cours de ses cinquante années d'existence. Les textes de Garon (2007-2008) et de Gagnon-Arguin (1992a, 1992b) constituent des historiques conventionnels qui rappellent I'ensemble des événements marquants de I'histoire de I'AAQ. Celui de Dugas se situe dans un contexte semblable. Quant à la Ligne $d u$ temps, elle constitue principalement une énumération des événements avec des explications sur certains d'entre eux. D'autres textes sont plus thématiques: les vingt premiers congrès, les mémoires et les prises de position, les activités de formation et de perfectionnement. Certains font appel à la mémoire de leur auteur, d'autres reposent sur l'exploitation des sources écrites, particulièrement la revue Archives et La Chronique. Tous ces textes mettent en lumière, de façon éclatante, le travail réalisé par les membres et leur engagement pour le rayonnement de l'AAQ ainsi que son implication dans la société.

\section{L'HISTOIRE DE L'AAQ DANS DES PUBLICATIONS EXTÉRIEURES}

La présentation de l'Association, de son histoire, de son organisation et de ses activités n'a fait l'objet que de peu d'articles dans d'autres revues, au Québec comme à l'extérieur. Toutefois, il faut signaler trois textes.

À l'occasion du 50 anniversaire de l'AAQ, deux périodiques en histoire consacrent un numéro spécial sur les archives et proposent un article sur l'Association. Histoire Québec, revue de la Fédération Histoire Québec, dans un numéro spécial intitulé «État des lieux de la mémoire archivistique», propose un article intitulé "L'Association des archivistes du Québec: une association en phase avec sa société », par Gilles Héon (Héon, 2017). L'auteur y rappelle les différents éléments des contextes politique, social et culturel qui ont favorisé la naissance de l'AAQ, les principales réalisations de l'Association en matière de perfectionnement, particulièrement ses publications d'ouvrages utiles à la gestion de I'information, de même que son implication dans les débats de société touchant la culture et la protection de l'information. En conclusion, il rappelle l'étroite collaboration interdisciplinaire qui existe entre les historiens et les archivistes dans le but de conserver et de mettre en valeur le patrimoine, et ce, chacun dans sa sphère de compétence. 
La revue Cap-aux-Diamants, revue d'histoire du Québec, présente aussi un numéro spécial sur les archives, sous le thème "Archives en tête ". Un des articles porte sur l'histoire de l'AAQ (Lévesque et Gagnon-Arguin, 2017). Les auteurs retournent au contexte de sa fondation et soulignent les activités associatives fondamentales que sont les publications et les activités de perfectionnement. Ils évoquent aussi les actions politiques et sociales à travers les mémoires présentés aux différents gouvernements en se portant à la défense de la culture et de l'histoire. Ils concluent en affirmant que I'AAQ a contribué, par l'engagement continu de ses membres, au développement de la discipline archivistique, à la reconnaissance des archives comme éléments de notre patrimoine, comme matériaux de recherche et comme soutien à la gestion des organisations.

II faut remonter à 1978 pour retrouver un article sur I'AAQ publié en dehors de ses propres publications. Celui-ci paraît dans la revue des archivistes belges, Archives et bibliothèque de Belgique (Héon, 1978). Intitulé "Association des archivistes du Québec», ce texte situe l'émergence de l'Association dans le contexte plus large de la Révolution tranquille et de ses répercussions sur les administrations. L'auteur présente ensuite l'histoire de I'AAQ et de son organisation, puis relève ses principaux défis comme la reconnaissance professionnelle et la formation institutionnelle; ses combats déterminants, dont «l'affaire des manuscrits»; la mise en place d'un lien institutionnel avec les archivistes canadiens par le Bureau canadien des archivistes et, enfin, le projet d'un autre lien avec les archivistes francophones. II conclut en affirmant sa confiance en cette association «jeune et fière » qui travaille au développement de la profession et de la discipline, et à la collaboration avec les archivistes d'ailleurs.

\section{L'AAQ ET LES RELATIONS INTERNATIONALES}

Sous le titre «Nos amitiés internationales», un texte paru en 1986 dans la revue Archives constitue une «chronologie des relations établies par l'Association des archivistes du Québec et ses membres avec des organismes et collègues étrangers, 1967-1985», comme l'indique son sous-titre (Héon, 1986). L'auteur y évoque les diverses activités qui ont marqué les relations de l'AAQ avec le Conseil international des archives (CIA) par la participation d'archivistes québécois à ses congrès et aux 
travaux de la Table ronde des archives ou par leur implication dans la section des associations professionnelles du CIA. Dans une partie «Éphémérides », il dresse ensuite une liste des déplacements d'archivistes québécois en dehors du Québec et d'archivistes de l'extérieur du Québec venus au pays. Puis, dans une partie consacrée aux publications, I'auteur recense les publications d'auteurs non québécois, surtout dans la revue Archives, et celles d'auteurs québécois à l'extérieur du Québec. II termine par une liste des participants au Stage international d'archives à Paris de 1968 à 1985. Ce rappel constitue un tableau impressionnant des déplacements des archivistes québécois, de leurs contacts avec différents milieux archivistiques internationaux et, par le fait même, des influences qu'ils ont partagées.

\section{L'AAQ SOUS LA LOUPE DE LA THÉORIE}

Deux textes intéressant également I'histoire de l'Association ont été écrits à l'aide d'approches théoriques pour analyser un phénomène. L'un traite du secrétariat administratif de l'AAQ dans une approche mémorielle, réalisé dans le cadre de travaux de maîtrise en histoire - archivistique de l'Université Laval (Héon, 1996). L'autre, tiré d'une recherche menée dans le cadre d'un doctorat en histoire, étudie l'AAQ à partir des modèles développés en sociologie des professions et des disciplines (GagnonArguin, 1992a).

\subsection{Information et mémoire}

Avec I'approche polysystémique de la mémoire utilisée par la professeure Martine Cardin dans le cadre de ses recherches, Gilles Héon met l'accent sur l'exploitation de l'information au secrétariat de I'Association. II analyse l'information dans ses dimensions matérielle (organisation des documents), fonctionnelle (place du secrétariat dans l'organisation) et symbolique (son rôle). Sous le volet Exploitation de l'information, il en décrit l'organisation (personnel, lieu), le système information (comment elle circule) et le système mémoire (essentielle, administrative et historique). Ce faisant, l'auteur scrute tous les angles de l'objet « archives» pour en faire ressortir toutes les significations, de leur caractère identitaire jusqu'aux impératifs de la recherche, mais surtout en privilégiant la reconnaissance du contexte de leur création. 


\subsection{Sociologie des professions et des disciplines}

La sociologie s'intéresse notamment aux développements des professions et des disciplines à travers leurs différentes manifestations. Les associations professionnelles en constituent des lieux privilégiés. C'est pourquoi l'étude de l'Association est intégrée dans une recherche menée dans le cadre d'études doctorales et publiée en 1992, où elle illustre le développement d'une profession et d'une discipline à travers ses effectifs et ses réalisations. (Gagnon-Arguin, 1992) Après l'avoir présentée comme association professionnelle, l'auteure en étudie l'effectif et le leadership, les activités et les publications. Un autre texte inspiré par la même approche décrit l'histoire de l'AAQ, son organisation administrative, son financement, les services à ses membres, ses rôles politique et social et ses valeurs professionnelles (Gagnon-Arguin, 1992a).

Toujours dans le même esprit, Louise Gagnon-Arguin prononce une conférence au 36 congrès de l'AAQ en 2006 (Gagnon-Arguin et Chouinard, 2006). Elle montre que les contextes politique, social, culturel et économique ainsi que le développement technologique du Québec entre 1960 et 2006 agissent comme toile de fond au développement de I'archivistique québécoise. Elle y présente ensuite les institutions d'archives selon les milieux, la profession d'archiviste, puis la mission, les tâches, la vie associative, l'image sociale de l'archiviste et enfin, I'archivistique comme discipline.

\section{CONCLUSION}

En reprenant I'histoire de I'Association des archivistes du Québec à partir des textes qui l'ont fait connaître, nous avons voulu mettre en lumière les différents points de vue des auteurs qui se sont intéressés à son histoire. Ces «histoires» écrites au fil des ans traduisent le vécu de l'époque où elles sont préparées; elles relatent des faits ou des événements peu après leur apparition et informent sur leur perception presque à chaud. Elles deviennent alors elles-mêmes des sources pour l'histoire de l'AAQ. Que ce soit à l'occasion d'anniversaires ou par un rappel chronologique d'activités internationales des membres ou de l'accueil de visiteurs étrangers, que ce soit sous l'angle de l'intérêt d'autres revues ou à travers des analyses en fonction de modèles théoriques, le rappel «des histoires» de l'AAQ constitue en soi « une nouvelle histoire » qui invite chacun à lire ou relire ces textes selon ses intérêts ou sa curiosité. 
Somme toute, I'histoire de l'AAQ a fait l'objet de plusieurs réflexions, études, analyses conduisant à la publication de rétrospectives diverses rappelant les manifestations de la vie des archivistes à travers leur association. Leur rappel chronologique et thématique, en résumant sommairement leur contenu, permet de mesurer les innombrables actions et réflexions menées depuis 50 ans. II s'agit donc, sous la forme d'un kaléidoscope, de l'histoire de l'AAQ à travers les textes.

LoUise Gagnon-Arguin

Professeure associée, École de bibliothéconomie et des sciences de l'information (EBSI), Université de Montréal.

\section{BIBLIOGRAPHIE CHRONOLOGIQUE}

\section{RÉFÉRENCES POUR L'HISTOIRE DE L'AAQ}

\section{4}

GARON, R. (1974). L'Association des archivistes du Québec. Archives, 74(2), 5-10.

\section{$\underline{1978}$}

GARON, R., MINOTTO C. et COUTURE C. (1978). L'Association des archivistes du Québec en 1978; bilan et perspectives, trois points de vue. Archives, 10(2), 57-67.

HÉON, G. (1978). Association des archivistes du Québec. Archives et bibliothèque de Belgique, XLIX(1-2), 195-200.

\section{2}

BEAUDIN, F. (1982). Née à Québec...mais conçue à Santa Fe, New Mexico (USA)... et à Québec! La Chronique, XI(4), 5.

CHOQUETTE, L. (1982). Félicitations à notre Association pour son

$15^{\mathrm{e}}$ anniversaire, le 9 décembre 1982. La Chronique, XI(4), 1-4. 
LESSARD, C. (1982). L'AAQ après quinze ans...; quelques propos d'un ancien président. La Chronique, XI(4), 6.

\section{6}

HÉON, G. (1986). Nos amitiés internationales ou Chronologie des relations établies par l'Association des archivistes du Québec et ses membres avec des organismes et collègues étrangers, 1967-1985. Archives, 17(4), 38-51.

\section{1}

LESSARD, C. (1991). Rappel historique sur les congrès de notre association. Trois-Rivières.

\section{2}

GAGNON-ARGUIN, L. (1992a). L'Association des archivistes du Québec. Dans L'archivistique, son histoire, ses acteurs depuis 1960. (124-178) Québec: Presses de I'Université du Québec.

GAGNON-ARGUIN, L. (1992b). L'Association des archivistes du Québec; vingt-cing ans d'histoire. Archives, 24(1-2), 9-46.

\section{6}

HÉON, G. (1996). L'exploitation de l'information au secrétariat administratif de l'Association des archivistes du Québec. Archives, 27(3), 11-27.

\section{6}

GAGNON-ARGUIN, L. et CHOUINARD, D. (2007). Les archivistes du Québec; placer la mémoire au cœur de l'action. Actes du $36^{\mathrm{e}}$ congrès de l'AAQ, Québec, 174-193.

\section{$\underline{2007}$}

BAILLARGEON, D. et LÉVESQUE, M. (2007-2008). L'Association des archivistes du Québec: une association impliquée, des archivistes engagés. Archives, 39(1), 43-92. 
GARON, L. (2007-2008). L'Association des archivistes: du congrès de 1988 à 2006. Archives, 39(1), 3-42.

NAHUET, R. (2007-2008). L'AAQ: la formation et le perfectionnement en archivistique. Archives, 39(1), 93-111.

\section{5}

GAGNON-ARGUIN, L. (2015). Carol Couture et la vie associative. Dans Gagnon-Arguin, L. et Lajeunesse, M. (dir.). Panorama de l'archivistique contemporaine: évolution de la discipline et de la profession; mélanges offerts à Carol Couture. (37-42) Québec: Presses de l'Université du Québec.

\section{$\underline{2017}$}

ASSOCIATION DES ARCHIVISTES DU QUÉBEC. (2017). Ligne du temps. Repéré à http://50ans.archivistes.qc.ca/ligne-temps-vie-association/

DUGAS, C. (2017). La Division de la gestion de documents et des archives de l'Université de Montréal et l'Association des archivistes du Québec: des chemins croisés. Archives, 46(2), 133-143.

HÉON, G. (2017). L'Association des archivistes du Québec: une association en phase avec sa société. Histoire Québec, 22(3), 5-7.

LÉVESQUE, M. et GAGNON-ARGUIN L. (2017). L'Association des archivistes du Québec, 1967-2017; 50 ans d'engagement professionnel et social. Cap-aux-Diamants, 131(automne), 4-6. 